\title{
AN EXTENSION OF A THEOREM OF KHAVINSON
}

\author{
JOHN FERRY
}

(Communicated by Paul S. Muhly)

\begin{abstract}
We extend a generalization of the Cauchy-Green formula, which in
\end{abstract} turn extends results in rational approximation.

Theorem. Let $K \subseteq \mathbb{C}$ be compact and have finite perimeter. Let $2<p<\infty$ and $f \in W^{1, p}(\mathbb{C})$. Then there exists a subset $E$ of $K$ satisfying $m_{2} E=0$ and

$$
\frac{1}{2 \pi i} \int_{B_{K}} \frac{f}{z-z_{0}} d z-\frac{1}{\pi} \iint_{K} \frac{\bar{\partial} f}{z-z_{0}} d A=\left\{\begin{array}{lll}
f\left(z_{0}\right) & \text { if } & z_{0} \in K \backslash E, \\
0 & \text { if } & z_{0} \in \mathbb{C} \backslash K .
\end{array}\right.
$$

Remarks. (1) $B_{K}$ is the reduced boundary of $K$, the "nice" portion of the topological boundary of $K$. For a precise definition of $B_{K}$ and of finite perimeter, see [2]. $W^{1, p}(\mathbb{C})$ is defined in [1]. $d m_{2}=d A$ denotes Lebesgue area measure and $\bar{\partial}$ indicates $\partial / \partial \bar{z}$.

(2) Khavinson [2, Theorem 2.1] proves the above for $f \in \operatorname{Lip}(1, \mathbb{C})$. The above theorem does extend Khavinson's; this will be discussed following the proof.

Proof. By Theorem 3.18 [1], there exists a sequence $\left(\phi_{n}\right)$ of $C^{\infty}$-functions having compact support, with $\phi_{n} \rightarrow f$ in $W^{1, p}(\mathbb{C})$. By Theorem 5.4 [1], $W^{1, p}(\mathbb{C})$ is continuously embedded in $\left(C \cap L^{\infty}\right)(\mathbb{C})$, the bounded continuous functions (with the sup norm).

$$
\text { Set } \begin{aligned}
F=\left\{z_{0} \in K: \int_{B_{K}} \frac{d|z|}{\left|z-z_{0}\right|}\right. & <\infty \text { and for each } n, \\
\phi_{n}\left(z_{0}\right) & \left.=\frac{1}{2 \pi i} \int_{B_{K}} \frac{\phi_{n}}{z-z_{0}} d z-\frac{1}{\pi} \iint_{K} \frac{\bar{\partial} \phi_{n}}{z-z_{0}} d A\right\},
\end{aligned}
$$

and $E=K \backslash F$. By Theorem 2.1 [2], $m_{2} E=0$.

Let $z_{0} \in \mathbb{C} \backslash E$. Since $\left(\phi_{n}\right)$ converges uniformly to $f$, we have

$$
\left|\int_{B_{K}} \frac{f-\phi_{n}}{z-z_{0}} d z\right| \leq\left\|f-\phi_{n}\right\|_{B_{K}} \int_{B_{K}} \frac{d|z|}{\left|z-z_{0}\right|} \longrightarrow 0,
$$

Received by the editors September 19, 1990.

1991 Mathematics Subject Classification. Primary 30E10, 31A10.

Key words and phrases. Cauchy-Green, rational approximation, finite perimeter, reduced boundary, Sobolev spaces, Lipschitz class. 
where $\|\cdot\|_{B_{K}}$ denotes the supremum over the set $B_{K}$. Writing $1 / p+1 / q=1$, we see

$$
\left|\iint_{K} \frac{\bar{\partial}\left(f-\phi_{n}\right)}{z-z_{0}} d A\right| \leq\left\|\bar{\partial}\left(f-\phi_{n}\right)\right\|_{L^{p}(K)}\left(\iint_{K} \frac{d A}{\left|z-z_{0}\right|^{q}}\right)^{1 / q} \longrightarrow 0 .
$$

Since $\phi_{n}\left(z_{0}\right) \rightarrow f\left(z_{0}\right)$, we are done. (By Theorem 2.1 [2], the above theorem holds for each $\phi_{n}$.)

Consider now the above theorem together with Theorem 2.1 [2]. The conclusions of both theorems involve only the values of the function in a neighborhood of $K$. By means of a cutoff function (multiply by a $C^{\infty}$-function having compact support, which is equal to 1 in a neighborhood of $K$ ), we can (in the hypotheses of the above theorems) replace the spaces $W^{1, p}(\mathbb{C})$ and $\operatorname{Lip}(1, \mathbb{C})$ by $W_{c}^{1, p}(\mathbb{C})$ and $\operatorname{Lip}_{c}(1, \mathbb{C})$, respectively, where the subscript $c$ refers to compact support. In the proof of Theorem 2.1 [2], Khavinson (basically) proves the inclusion $\operatorname{Lip}_{c}(1, \mathbb{C}) \subseteq W_{c}^{1, \infty}(\mathbb{C})$. Clearly $W_{c}^{1, \infty}(\mathbb{C}) \varsubsetneqq W_{c}^{1, p}(\mathbb{C})$ for $2<p<\infty$.

It is in this manner that the above theorem is an extension of Khavinson's.

Using the above theorem, we may extend Theorem 3.1, Theorem 3.2, and Corollary 3.1 of [2] to functions in $W^{1, p}(\mathbb{C})$. The statements and proofs are otherwise identical: simply replace $f \in \operatorname{Lip}(1, \mathbb{C})$ by $f \in W^{1, p}(\mathbb{C})$.

\section{ACKNOWLEDGMENT}

I wish to thank Xiao Li for finding a mistake in my original proof of the theorem and for bringing the paper [2] to my attention

\section{REFERENCES}

1. R. Adams, Sobolev spaces, Academic Press, Orlando, FL, 1975.

2. D. Khavinson, The Cauchy-Green formula and rational approximation on the sets with a finite perimeter in the complex plane, J. Funct. Anal. 64 (1985), 112 - 123.

Department of Mathematics, Virginia Polytechnic Institute and State University, BLACKSBURG, VIRGINIA 2406' 